\title{
Comparison of Pannexin1 ATP Channel Expression in Rat Brain Tissue
}

\author{
A.C. Cone*, D. Boassa*, K. Fuller*, M.E. Martone*\#, and G.E. Sosinsky*\# \\ * National Center for Microscopy and Imaging Research and \# Department of Neurosciences, \\ University of California San Diego, 9500 Gilman Drive MC 0608, La Jolla, CA 92093-0608
}

The pannexin proteins were originally speculated to have gap junction-like function based on their predicted structure that includes a "connexin-like fold". However subsequent research, including results from our laboratory, have indicated that they do not function as intercellular channels, but instead function as single membrane channels in vivo.

Pannexin1 (Panx1) is the most characterized of the three pannexins and functions to release cytosolic ATP. It is highly expressed in many tissues including the immune system, heart, kidney and the central nervous system. Panx1 has been shown to be a component of the neuronal inflammasome [1] and plays a critical role in ATP release in astrocytes [2]. However, expression studies done by in situ hybridization and by immunolabeling approaches in rodent brain tissue do not totally coincide and subcellular localizations in tissue have been ambiguous [3,4]. Comparison of protein labeling and gene expression is complicated in the nervous system, because gene expression primarily occurs in the cell bodies while proteins may be anywhere in the cell. Thus, comparison of the results requires knowledge of where the processes of neurons are located and their connectivity. Sources of variability in protein labeling experiments include differences between labeling patterns produced by various antibodies for Panx 1, species differences, and protocol differences.

Here we compare and contrast the labeling patterns of four Panx1 antibodies in rat brain tissue using western blot analysis and immunocytochemistry. We compared overlapping patterns of three polyclonal antibodies (rabbit or chicken) and one monoclonal antibody. Myc tagged Panx1 exogenously expressing HeLa cells were used as a positive control for each antibody. Each of the four Panx1 antibodies was generated against different epitopes. The monoclonal antibody, mouse anti-Panx1 \#503 developed by our laboratory, recognizes the N-terminus at amino acids 17-31. Two different polyclonal chicken-anti Panx1 antibodies were used. The commercially available Diatheva chicken anti-Panx1 antibody recognizes the first intracellular loop at amino acids 135-165, while the chicken anti-Panx1 \#4515 antibody from Gerhard Dahl [5], recognizes the C-terminus at amino acids 414-425. A polyclonal rabbit anti-Panx1 antibody that we developed (denoted as \#57) recognizes amino acids 148-164, an epitope overlapping with that of the Diatheva chicken antibody.

We performed high-resolution localizations of Panx 1 in brain slices using automated wide-field mosaic confocal imaging such that multiple overlapping image stacks are acquired and assembled into one large image (Fig. 1A). The mosaic can be browsed at multiple resolutions including the entire specimen, regions of interest, certain cell populations, and cellular compartments (at maximum resolution). The ability to combine gross structural imaging with high-resolution microscopy on a large scale significantly advances our ability to detect and analyze multiple brain regions across this heterogeneous organ. This imaging strategy provides us with a good approach for comparing different patterns of Panx 1 expression over extensive regions of rat brain.

Although these four anti-Panx1 antibodies perform similarly in labeling cultured cells both in western blots and immunofluorescence, there were significant differences between expression 
patterns in brain tissue (Fig. 1B-E). These are all intracellular epitopes and their accessibilities in a complex tissue like the brain may account for differences in labeling patterns, however, Panx1 has recently been shown to contain a caspase cleavage site that could cause differential recognition of the cleaved protein by these four antibodies [6]. Thus, the complex labeling patterns we see may be a reflection of different cellular and subcellular localizations where each antibody is highlighting unique and accessible Panx1 populations.

\section{References}

[1] W.R. Silverman et al., J Biol Chem 284 (2009) 18143-51.

[2] R. Iglesias et al., J Neurosci 29 (2009) 7092-7097.

[3] E.S. Lein et al., Nature 445 (2007) 168-172.

[4] G. Zoidl et al., Neuroscience 146 (2007) 9-16.

[5] S. Lacovei et al., Proc Natl Acad Sci 103 (2006) 7655-9.

[6] F.B. Checkeni et al., Nature 467 (2010) 863-7

[7] Supported by NIH grants GM072881 (GS), GM092457 (AC), DA016602 (MM), AHA Grant 10SDG2610281 (DB), RR004050 (Mark Ellisman) NSF grant MCB-0543934.

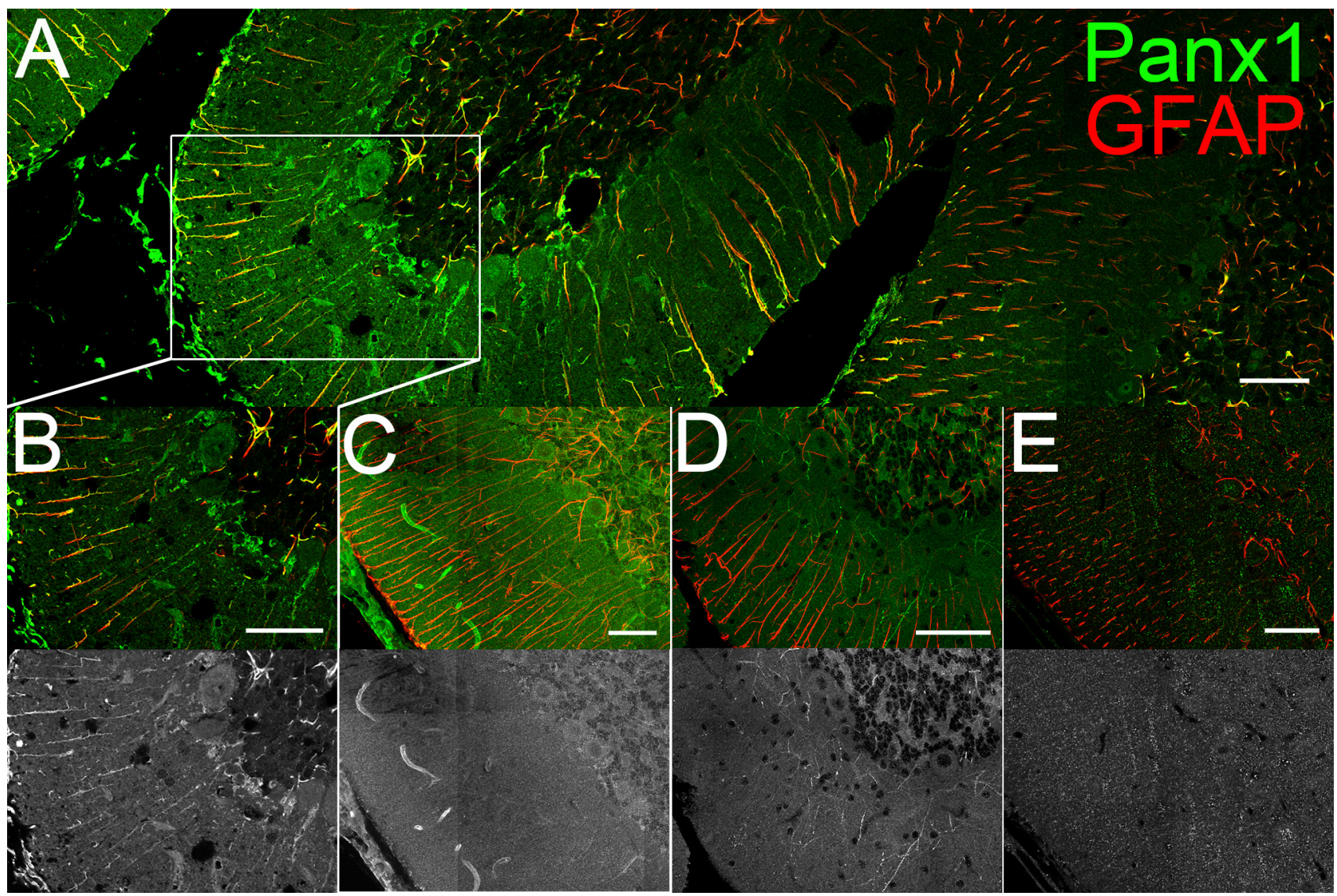

FIG. 1. A. Mosaic of 15 individual fields digitally stitched together to create a single, high-resolution image within a sagittal section of rat cerebellum labeled with Diatheva chicken anti-Panx 1 antibody. Anti-Panx1 antibodies are green and astrocytes are labeled using guinea pig anti-GFAP (red). B-E. Views of molecular layer and Purkinje cell layer from mosaics made with: B. Diatheva chicken antiPanx1 antibody, C. chicken anti-Panx1 \#4515, D. mouse anti-Panx1 \#503, and E. rabbit anti-Panx1 $\# 57$. Lower panels are grayscale images of Panx1. Scale bars $=50$ microns 